\title{
Langmuir
}

pubs.acs.org/Langmuir

(C) 2010 American Chemical Society

\section{PEGylated Phospholipid Membrane on Polymer Cushion and Its Interaction with Cholesterol}

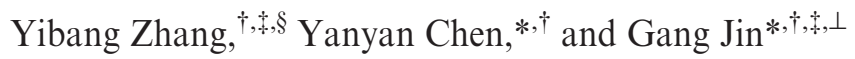 \\ ${ }^{\dagger}$ Suzhou Institute of Nano-Tech and Nano-Bionics, Chinese Academy of Sciences, Suzhou, Jiangsu 215125, P. R. \\ China, ${ }^{\sharp}$ Institute of Biophysics, Chinese Academy of Sciences, Beijing 100101, P. R. China,${ }^{\S}$ Graduate University \\ of Chinese Academy of Sciences, Beijing 100049, P. R. China, and ${ }^{\perp}$ Institute of Mechanics, Chinese Academy of \\ Sciences, Beijing 100190, P. R. China
}

Received March 10, 2010. Revised Manuscript Received April 15, 2010

\begin{abstract}
By employing poly(ethylene glycol) (PEG) shielding and a polymer cushion to achieve air stability of the lipid membrane, we have analyzed PEG influence on dried membranes and the interaction with cholesterol. Small unilamellar vesicles (SUVs) formed by the mixture of 1,2-dimyristoylphosphatidylcholine (DMPC) with different molar fraction of 1,2-distearoyl-sn-glycero-3-phosphoethanolamine- $N$-[methoxy(poly(ethylene glycol))-2000] (DSPE-PEG 2000 ) adsorb and fuse into membranes on different polymer-modified silicon dioxide surfaces, including chitosan, poly(L-lysine) (PLL), and hyaluronic acid. Dried membranes are further examined by ellipsometer and atomic force microscopy (AFM). Only chitosan can support a visible and uniform lipid array. The thickness of dry PEGylated lipid membrane is reduced gradually as the molar fraction of PEG increases. AFM scanning confirms the lipid membrane stacking for vesicles containing low PEG, and only a proper amount of PEG can maintain a single lipid bilayer; however, the air stability of the membrane will be destroyed if overloading PEG. Cholesterol incorporation can greatly improve the structural stability of lipid membrane, especially for those containing high molar fraction of PEG. Different amounts of cholesterol influence the thickness and surface morphology of dried membrane.
\end{abstract}

\section{Introduction}

SUVs $^{1}$ are widely used for the preparation of supported phospholipid bilayers (SPB), which have been used as model membranes to study cell recognition, ${ }^{2}$ membrane proteins, or ion channel-based biosensors. ${ }^{3}$ However, SPB systems will be quickly destroyed upon exposure to the air-water interface and therefore have to remain underwater when applying research tools such as AFM. ${ }^{4-7}$ Recently, a few published research works have focused on creating air-stable phospholipid membranes; nevertheless, there are still some problems, such as reduced fluidity, ${ }^{8}$ high density protein coverage, ${ }^{9}$ or need of careful handling, ${ }^{10}$ which make these lipid membranes unsuitable for biosensor applications. ${ }^{11}$ In addition, fluorescence labeling and microscopy ${ }^{9-12}$ are mainly used in these studies, which cannot tell the detailed structure of dried phospholipid membrane. In an effort to solve these problems, we recently have designed a polymer surrounding phospholipid membrane on silicon wafer.

*Corresponding authors. E-mail: yychen2006@sinano.ac.cn (Y.C.),gajin@ imech.ac.cn (G.J.).

(1) Tamm, L. K.; McConnell, H. M. Biophys. J. 1985, 47, 105-113.

(2) McConnell, H. M.; Watts, T. H.; Weis, R. M.; Brian, A. A. Biochim. Biophys.

Acta 1986, 864, 95-106.

(3) Edward, T. C.; Cremer, P. S. Surf. Sci. Rep. 2006, 61, 429-444.

(4) Reviakine, I.; Brisson, A. Langmuir 2000, 16, 1806-1815.

(5) Ralf, P. R.; Brisson, A. R. Biophys. J. 2005, 88, 3422-3433.

(6) Tero, R.; Takizawa, M.; Li, Y. J.; Yamazaki, M.; Urisu, T. Langmuir 2004, 20,7526-7531.

(7) Charrier, A.; Thibaudau, F. Biophys. J. 2005, 89, 1094-1101.

(8) Eric, E. R.; Bruce, B.; Tony, S.; John, C. C.; David, F. O.; Saavedra, S. S. Langmuir 2001, 17, 2305-2307.

(9) Holden, M. A.; Jung, S.-Y.; Yang, T.; Castellana, E. T.; Cremer, P. S. J. Am. Chem. Soc. 2004, 126, 6512-6513.

(10) Deng, Y.; Wang, Y.; Holtz, B.; Li, J.; Traaseth, N.; Veglia, G.; Stottrup, B. J.; Elde, R.; Pei, D.; Guo, A.; Zhu, X.-Y. J. Am. Chem. Soc. 2008, 130, 62676271 .

(11) Albertorio, F.; Diaz, A. J.; Yang, T.; Chapa, V. A.; Kataoka, S.; Castellana,

E. T.; Cremer, P. S. Langmuir 2005, 211, 7476-7482.

(12) Vist, M. R.; Davis, J. H. Biochemistry 1990, 29, 451-464.
By employing PEG shielding and chitosan cushion for achieving the air stability of lipid membrane, we have examined the structure information on dried phospholipid membrane and their morphological details with AFM.

Cholesterol is required for viability and cell proliferation, and it is known that cholesterol modulates the fluidity and increases the structural order of the phospholipids hydrocarbon chains of cell membranes. ${ }^{12}$ There have been a lot of researches and reviews on the interaction between cholesterol and phospholipids membranes. ${ }^{13-22}$ Recently, published works have shown cholesterol derivatives can be used as tethers for maintaining air stability of lipid membrane. ${ }^{10,23}$ In our experiments, cholesterol is inserted into PEGylated phospholipid membrane to examine their potential interactions. Ellipsometer and AFM are combined together to observe the condensing and ordering effect of cholesterol on lipid membranes.

\section{Experimental Section}

Materials. Aminopropyltriethoxysilane (APTES), glutaraldehyde, PLL, and cholesterol were purchased from Sigma. DMPC

(13) Bhattacharya, S.; Haldar, S. Biochim. Biophys. Acta 2000, 1467, 39-53.

(14) Dahl, C. D. J. Biology of Cholesterol; CRC Press: Boca Raton, FL, 1988.

(15) Finean, J. B. Chem. Phys. Lipids 1990, 54, 147-156.

(16) Khizar, H. S.; Hugo, K. C.; Richard, J. B.; Evans, S. D. J. Phys. Chem. B 2007, 111, 379-386.

(17) McConnell, H. M.; Radhakrishnan, A. Biochim. Biophys. Acta 2003, 1610, 159-173.

(18) Ohvo-Rekilä, H.; Ramstedt, B.; Leppimäki, P.; Peter, S. J. Prog. Lipid Res. 2002, 41, 66-97.

(19) PL, Y. Cholesterol in Membrane Models; CRC Press: Boca Raton, FL, 1993.

(20) Radhakrishnan, A.; McConnell, H. M. J. Am. Chem. Soc. 1999, 121, 486-487.

(21) Radhakrishnan, A.; McConnell, H. M. Biophys. J. 1999, 77, 1507-1517.

(22) Yeagle, P. L. Biochim. Biophys. Acta 1985, 822, 267-287.

(23) Blasi, L.; Pisignano, D.; Di Benedetto, F.; Maruccio, G.; Ciccarella, G.;

Maffei, A.; Vasapollo, G.; Cingolani, R.; Rinaldi, R. Biochim. Biophys. Acta 2005, 1714, 93-102. 
PEGylated phospholipid membrane

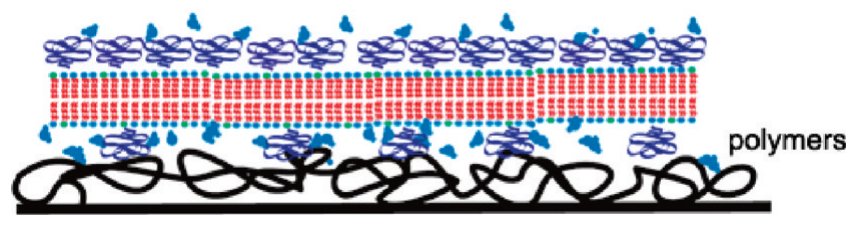

Figure 1. Schematic polymer cushioned phospholipid bilayers. Both polymer cushions and PEG shielding share the important factors for air-stable lipid bilayers formation.

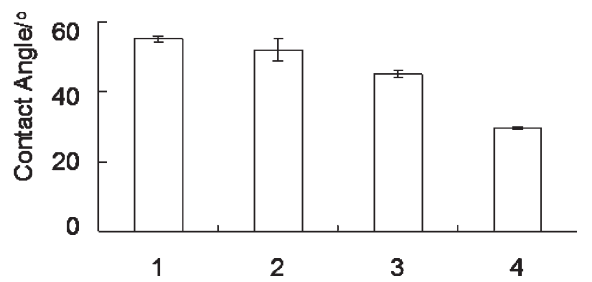

Figure 2. Water contact angle measurement of silicon dioxide surface after different modification: 1, APTES; 2, LMW chitosan; 3, PLL; 4, HA.

and DSPE-PEG 2000 were purchased from Avanti Polar Lipid, Inc. (Alablaster, AL). Low molecular weight chitosan (MW: $10000,85 \%$ deacetylation) was obtained from Haidebei Marine Bioengineering Co. (Jinan, Shandong, China). Hyaluronic acid (MW: 60 000) was provided by Freda Biochem. Co. Ltd. (Jinan, Shandong, China). $N$-Hydroxysuccinimide (NHS) and $N$-ethyl$N^{\prime}$-(3-diethylaminopropyl)carbodiimide (EDC) were purchased from Acros Organics, Belgium.

Substrates Surface Modification. Polished silicon wafers were cleaned with piranha solution $\left(98 \% \mathrm{H}_{2} \mathrm{SO}_{4}: 30 \% \mathrm{H}_{2} \mathrm{O}_{2}=\right.$ $3: 1, \mathrm{v} / \mathrm{v}$ ) and further modified with APTES according to the protocols described before. ${ }^{24}$ Glutaraldehyde $(5 \%)$ was used as cross-linker for coupling LMW chitosan $(10 \mathrm{mg} / \mathrm{mL})$ or PLL $(5 \mathrm{mg} / \mathrm{mL})$ onto the aminated silicon dioxide surface. NHS/EDC $(50 \mathrm{mM} / 200 \mathrm{mM})$ mixture was used as carboxyl activator to bind HA ( $1 \mathrm{mM})$ onto the APTES-treated silicon wafer.

Liposome Preparation. SPB was formed through the extruded SUVs fusion method. Briefly, the desired mole fractions of DSPE-PEG $_{2000}$ and cholesterol were mixed with DMPC in chloroform. The solvent was then evaporated under a stream of nitrogen followed by desiccation under vacuum for at least $2 \mathrm{~h}$. The thin lipid film on the wall of vial was rehydrated in PBS (137 mM NaCl, $2.7 \mathrm{mM} \mathrm{KCl}, 10 \mathrm{mM} \mathrm{Na}_{2} \mathrm{HPO}_{4}$ and $2 \mathrm{mM}$ $\mathrm{KH}_{2} \mathrm{PO}_{4}$, pH 7.4) solution to a concentration of $1 \mathrm{mM}$ DMPC. The solution was clarified by a water bath ultrasonic cleaner and followed by being extruded through a polycarbonate filter, which had an average pore size of $50 \mathrm{~nm}$. A sufficient amount of each SUVs solution was dotted onto the functionalized surface and incubated for $15 \mathrm{~min}$ at room temperature. Excess vesicles were removed from the surface by rinsing with copious amounts of ultrapure water. The substrate was dried with pure nitrogen gas before further detection.

Water Contact Angle Measurements. The static water contact angles of modified silicon slides were measured on a Dataphysics OCA20 contact-angle system (Dataphysics, Germany) at room temperature. Silicon slides were rinsed with ultrapure water and dried under nitrogen gas before each contact angle measurement.

Imaging Ellipsometry. The detection of phospholipid membrane was carried out on an homemade imaging ellipsometer. ${ }^{25,26}$

(24) Wang, Z. H.; Jin, G. J. Immunol. Methods 2004, 285, 237-243.

(25) Jin, G.; Pentti, T.; Ingemar, L.; Arwin, H. Anal. Biochem. 1995, 232, 69-72.

(26) Jin, G. Phys. Status Solidi A 2008, 205, 810-816.

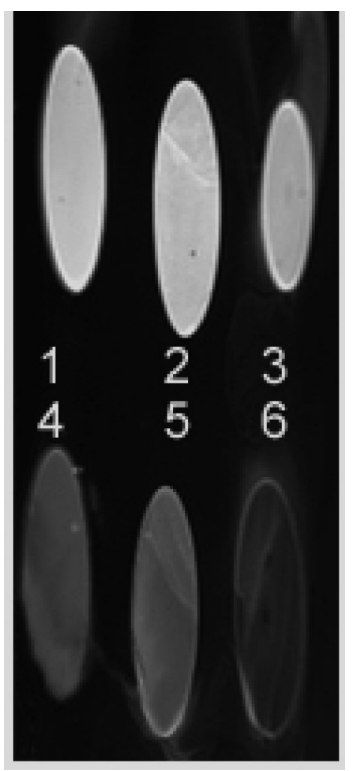

Figure 3. Ellipsometric image of air-stable chitosan-cushioned PEGylated lipid membrane. The numbers indicate different molar ratios of DSPE-PEG ${ }_{2000}$, which are $0.1,0.25,0.5,1.5,2.5$, and $5 \mathrm{~mol} \%$, respectively.

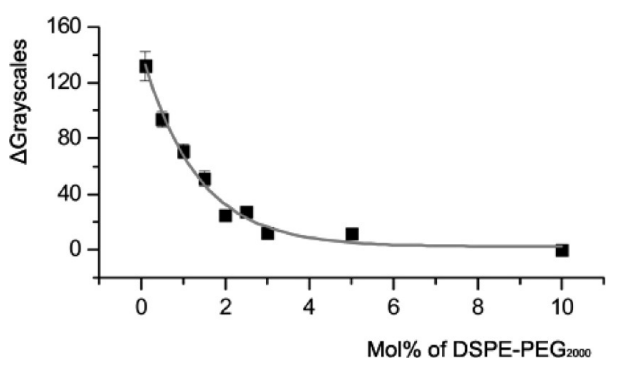

Figure 4. Grayscales values of lipid membrane against mole fraction of DSPE-PEG 2000 .

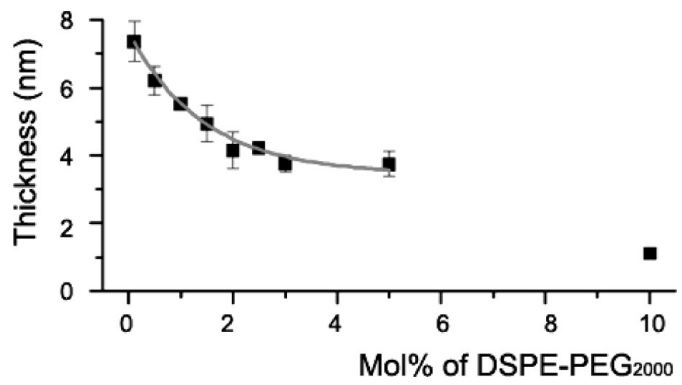

Figure 5. Phospholipid membrane thickness against mole fraction of DSPE-PEG 2000 .

The ellipsometer used was an enhanced standard single-beam model, which combined the power of ellipsometry with microscopy and worked in the off-null mode. It may visualize thin layer with image in grayscales, and the value of grayscales is proportional to molecule mass surface concentration or membrane thickness; i.e., higher grayscale values stand for more mass densities or membrane thickness. Otherwise, the thickness of lipid layer was measured by variable angle spectroscopy ellipsometer (Wollam M2000DI). Incident angles were fixed at $65^{\circ}, 70^{\circ}, 75^{\circ}$, and $80^{\circ}$.

AFM Characterization. All AFM images were taken using a commercially available instrument (Veeco; MultiMode III). A tapping mode cantilever with an oscillation frequency of 

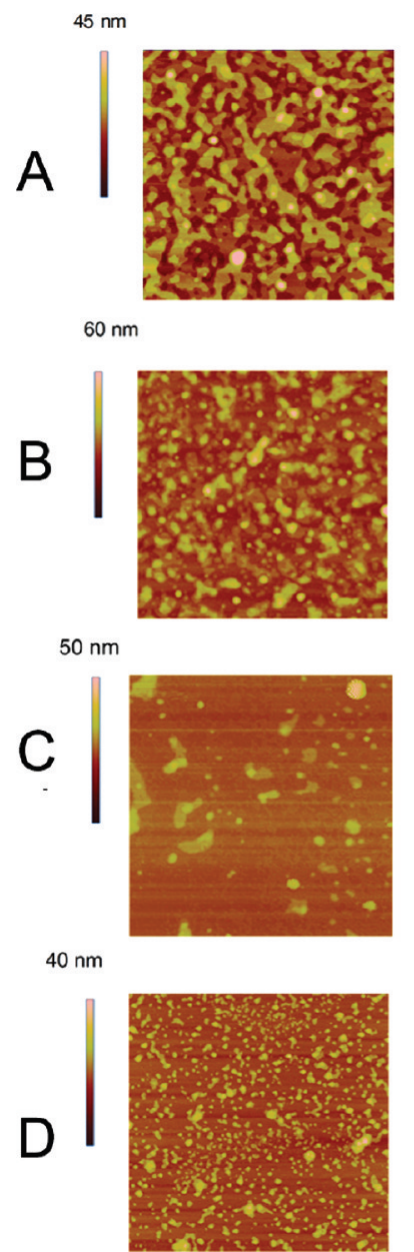

$30 \mathrm{~nm}$

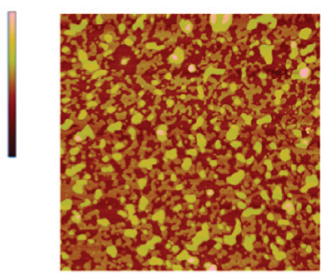

$40 \mathrm{~nm}$

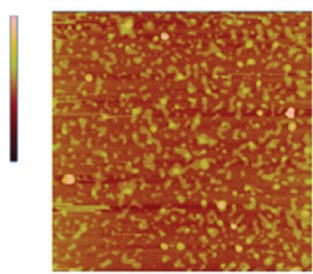

$20 \mathrm{~nm}$

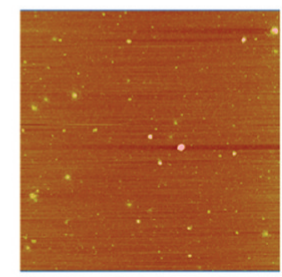

$20 \mathrm{~nm}$

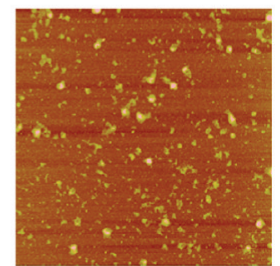

$20 \mathrm{~nm}$

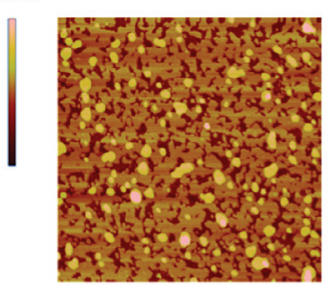

$50 \mathrm{~nm}$

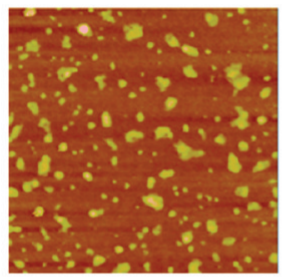

$20 \mathrm{~nm}$

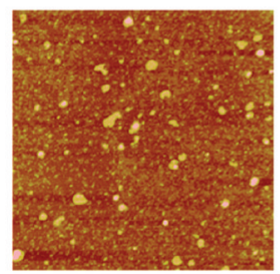

$20 \mathrm{~nm}$

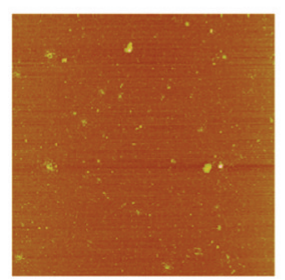

$20 \mathrm{~nm}$

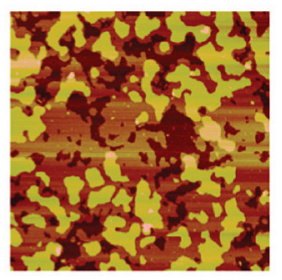

$50 \mathrm{~nm}$

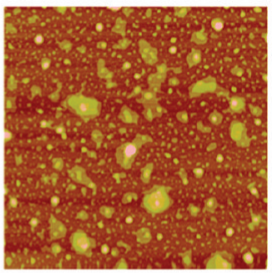

$30 \mathrm{~nm}$

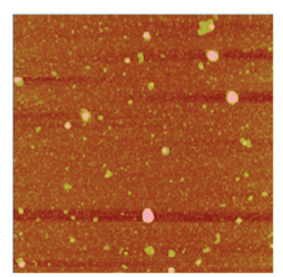

$20 \mathrm{~nm}$

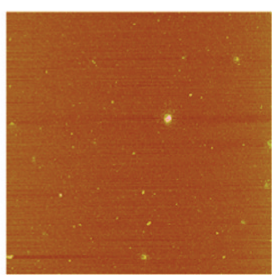

Figure 6. AFM characterization of chitosan cushioned lipid membrane containing (A) $0.5 \mathrm{~mol} \%$ DSPE-PEG 2000 , (B) $1.5 \mathrm{~mol} \%$ DSPE$\mathrm{PEG}_{2000}$, (C) $3 \mathrm{~mol} \% \mathrm{DSPE}_{-\mathrm{PEG}} \mathrm{D}_{2000}$, and (D) $5 \mathrm{~mol} \% \mathrm{DSPE}_{-} \mathrm{PEG}_{2000}$. The amount of cholesterol added is $0,5,10$, and $30 \mathrm{~mol} \%$ from left to right. Scan size is $5 \times 5 \mu \mathrm{m}^{2}$.

$200-300 \mathrm{kHz}$ was used during the procedure. AFM imaging was performed in air with no environmental controls.

\section{Results and Discussion}

It was reported that either $\mathrm{PEG}^{11}$ or $\gamma$-aminopropylsilane $\mathrm{e}^{27,28}$ could enhance the air stability of lipid membrane; unfortunately, in our pre-experiments, DMPC/DSPE-PEG 2000 membrane could be easily removed from APTES-modified silicon wafer after vigorous washing and nitrogen gas drying. Neither grayscales addition nor thickness change was detected by ellipsometry. We have coated three different kinds of oligosaccharides or polymers, i.e., LMW chitosan, PLL, and hyaluronic acid, on the silicon wafer as lipid membrane support (Figure 1). The water static contact angles of silicon wafers after different surface modification are shown in Figure 2. However, only LMW chitosan cushions give a visible lipid membrane array, seen in Figure 3, which is confirmed through AFM scanning.

Imaging ellipsometry may visualize thin layers with grayscale image, and the value of grayscale is proportional to molecule mass surface concentration or membrane thickness; ${ }^{26}$ i.e., higher grayscale values stand for more mass densities or membrane thickness. We have analyzed the relationship between DSPE$\mathrm{PEG}_{2000}$ molar fraction and $\Delta$ Grayscales or thickness of the membrane, which is shown in the plots of Figures 4 and 5. The

(27) Fang, Y. J. Am. Chem. Soc. 2006, 128, 3158-3159.

(28) Fang, Y.; Frutos, A. G.; Lahiri, J. J. Am. Chem. Soc. 2002, 124, 2394-2395.
$\Delta$ Grayscales or thickness of PEGylated lipid membrane is reduced gradually while the molar fraction of DSPE-PEG 2000 increased. SUVs containing DSPE-PEG 2000 below $1 \mathrm{~mol} \%$ will greatly enhance the membrane thickness, which is caused by bilayer aggregates and/or unfused vesicles. In the AFM photos accordingly, SUVs containing $0.5 \mathrm{~mol} \% \mathrm{DSPE}_{\mathrm{PEG}} \mathrm{D}_{200}$ form multiple lipid bilayers while 3 mol \% DSPE-PEG 2000 give us relatively smooth single lipid bilayers on chitosan cushion.

It is interesting that PEGylated lipids insert into the membrane and play roles as regulators during the SUVs fusion process and membrane formation. AFM scanning photos in Figure 6 have confirmed this. When PEGylated lipids are in a lower density, suspended vesicles can adhere or deposit onto formed lipid bilayers due to van der Waals attraction, ${ }^{19-21}$ resulting in multiple layers formation. If the hydration repulsion or stealthy effect ${ }^{29}$ between vesicles and membrane is improved as DSPE-PEG 2000 concentration is raised, the vesicles in the solution may cease to adsorb onto the existing lipid membrane and a single bilayer is achieved.

But it is too early to say the air-stable lipid bilayer can be formed on the chitosan cushions only if the DSPE-PEG 2000 is added. We find lipid membrane become scattered debris in air when $5 \mathrm{~mol} \%$ DSPE $\mathrm{PEG}_{2000}$ is added. When DSPE-PEG 2000 reaches as high as $10 \mathrm{~mol} \%$, the membrane is totally washed away, although the SUVs may have fused into membrane under

(29) Lasic, D. D.; Needham, D. Chem. Rev. 1995, 95, 2601-2628. 


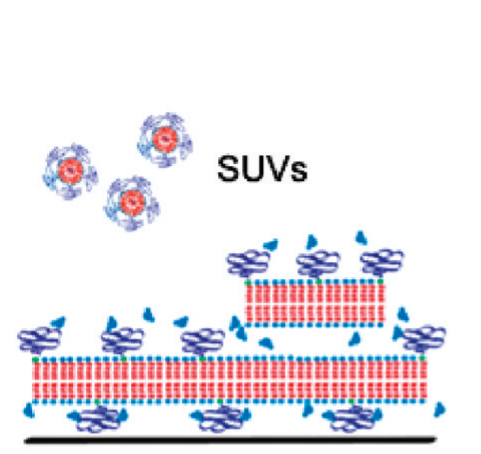

a)

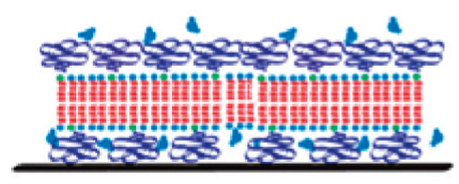

b)
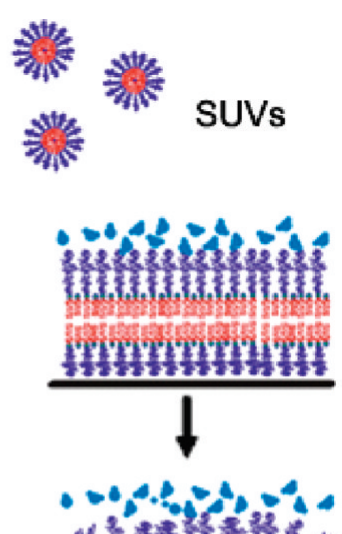

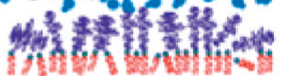
Hath

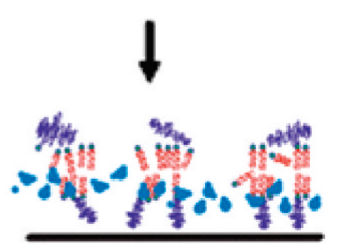

c)

Figure 7. Mechanism illustration of PEG induced phospholipid bilayers with different structure and washing stability. (a) Multiple lipid bilayers formed by low DSPE-PEG 2000 content SUVs. (b) Single lipid bilayers containing a proper density of PEG moieties. (c) Air and fluid instability caused by overloading DSPE-PEG 2000 .

water before treatment. The grayscales addition is beyond the detection limit, and the membrane thickness decreases to $1.6 \mathrm{~nm}$ only. As per PE-PEG 2000 could bind more than 210 water molecules, ${ }^{30}$ the interactions between bulky hydrophilic polymers and water molecules might serve one possible reason for membrane collapse. Steric hindrance among PEG brushes is also responsible for the instability of membrane when PEGylated lipids are in high density. It has been recognized that when the concentration of grafted PEG polymers is increased, PEG-PEG interaction will perturb the surface and cause a transition from liposome to micelle. ${ }^{31}$ Under a vigorous or repeated physically external force, some hydrophilic PEG molecules may be pulled out from lipid structure, inducing the deformation and whole depletion of lipid membrane in the end (possible mechanism is illustrated in Figure 7). In a later real-time QCM-D analysis, we find no frequency or dissipation reduction for high PEGylated lipid membrane during the rinsing with PBS and water. As a result, we conclude the membrane removal very possibly happened when it is undergoing desiccation treatment with nitrogen gas.

In biological systems, it has long been known that the incorporation of cholesterol into phospholipid membrane provokes their ordering and condensing. The fatty acyl chain of the phospholipids is flexible and is able to complement perfectly the shape of cholesterol such that the number of hydrophobic contacts is high and the packing is tight. ${ }^{32,33}$ The amount of cholesterol may vary with the type of membrane; too high level of

(30) Tirosh, O.; Barenholz, Y.; Katzhendler, J.; Priev, A. Biophys. J. 1998, 74, 1371-1379.

(31) Needham, D.; McIntosh, T. J.; Lasic, D. D. Biochim. Biophys. Acta 1992, $1108,40-48$.

(32) Han, J. H.; Taylor, J. D.; Phillips, K. S.; Wang, X.; Feng, P.; Cheng, Q. Langmuir 2008, 24, 8127-8133.

(33) Katarzyna, H. W.; Patrycja, D. Ł. J. Phys. Chem. B 2008, 112, 11324-11332.

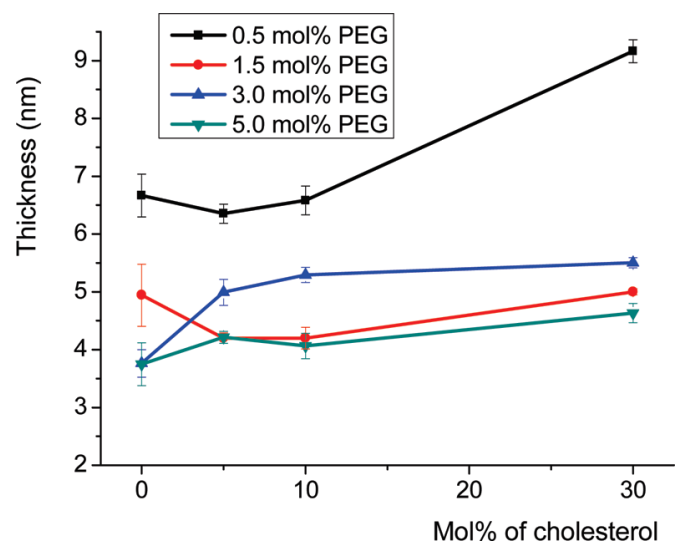

Figure 8. Plots of PEGylated membrane thickness versus adding molar fraction of cholesterol. Lipid membrane containing DSPE-PEG $20000.5 \mathrm{~mol} \%$ (black square), $1.5 \mathrm{~mol} \%$ (red circle), $3.0 \mathrm{~mol} \%$ (blue triangle), and $5.0 \mathrm{~mol} \%$ (green triangle). Error bar stands for the standard deviation calculated from at least six separate measurements.

cholesterol will cause cells to be too rigid and further the blood pressure problems, whereas decreased amounts of sterol in the membrane increase glucose and ions permeability. ${ }^{34,35}$ It has been reported that cholesterol incorporated into phospholipid membrane has a dynamic effect as its molar fraction changes: ${ }^{36}$ 10-30 mol \% cholesterol mixed lipid layer has densely packed and ordered structures, but the $50 \mathrm{~mol} \%$ cholesterol induces membrane structures irregular and disordered. As a result, the molar ratio of cholesterol in our PEGylated phospholipid membrane is limited not more than $30 \mathrm{~mol} \%$. As described above, we find when DSPE-PEG 2000 is in a relatively low molar fraction, vesicles in the suspension continue to absorb or deposit onto formed phospholipid membrane and fuse into multiple bilayers. However, cholesterol incorporation into lipid membranes influences their total thickness a little after being analyzed by spectrometric ellipsometry, which is shown in Figure 8. Noting that as low as $5 \mathrm{~mol} \%$ of cholesterol reduces the thickness of lipid membrane containing $1.5 \mathrm{~mol} \%$ DSPE-PEG 2000 , we suspect that cholesterol might play a disordering role by reducing the interaction between hydrocarbon tails of lipids and also the hydrophilic heads between neighboring layers. At different concentration level of cholesterol from 0 to $30 \mathrm{~mol} \%$, lipid membranes containing $1.5-5 \mathrm{~mol} \% \mathrm{DSPE}_{-} \mathrm{PEG}_{2000}$ restrict their thickness within the $4-5.5 \mathrm{~nm}$ range except the membrane with $0.5 \mathrm{~mol} \%$ DSPE-PEG $_{2000}$, although some detailed surface morphological differences under AFM tips exist. Especially, for the membrane with $5 \mathrm{~mol} \%$ PEGylated lipids, the air instability is improved dramatically after cholesterol addition, which can serve as a direct proof for cholesterol's condensing function. Herein, we can see the cooperation roles of PEG and cholesterol in the formation of smooth and dense air-stable single phospholipid bilayer.

Considering the membrane condensing effect of cholesterol, we also examine its power on lipid membrane at the PLL-modified surface. The ellipsometric characterization suggests that cholesterol addition may elevate the membrane thickness (from less than 2 to $4 \mathrm{~nm}$ or above depending on the DSPE-PEG 2000 and cholesterol molar fraction). Their characterization by AFM is shown in Figure 9. Doping of cholesterol can protect those lipid membranes supported by PLL from dehydration damage,

(34) Demel, R. A.; Dekruyff, B. Biochim. Biophys. Acta 1976, 457, 109-132.

(35) Heiniger, H. J.; Kandutsch, A. A.; Chen, H. W. Nature 1976, 263, 515-517.

(36) Kwangmeyung, Kim; Chulhee, Kim; Byun, Y. Langmuir 2001, 17, 5066- 

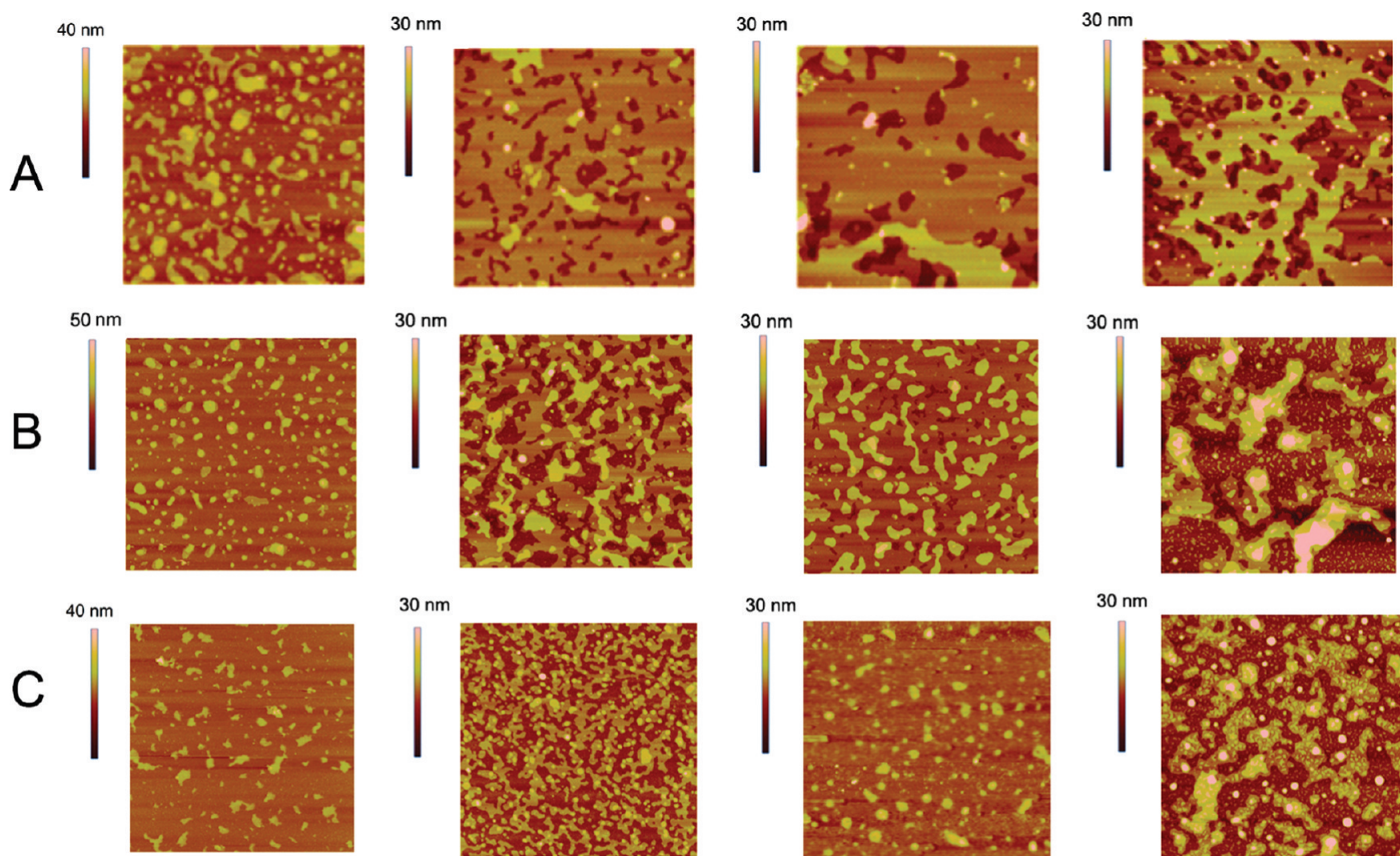

$30 \mathrm{~nm}$

\section{$30 \mathrm{~nm}$}
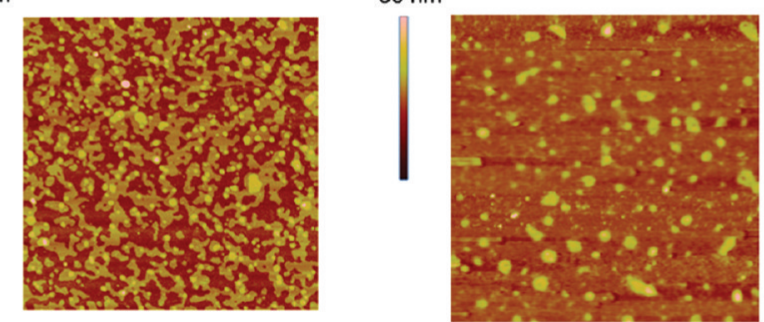

$30 \mathrm{~nm}$

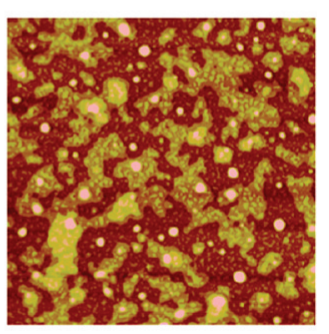

$50 \mathrm{~nm}$

$30 \mathrm{~nm}$

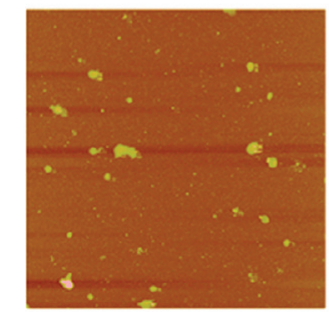

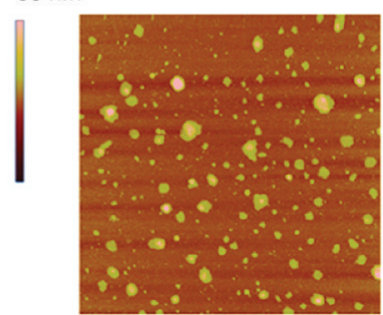

$30 \mathrm{~nm}$

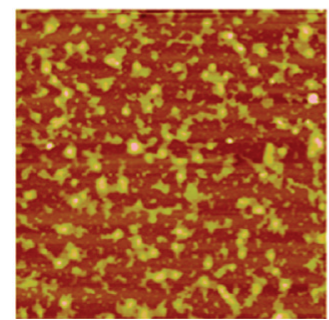

$20 \mathrm{~nm}$

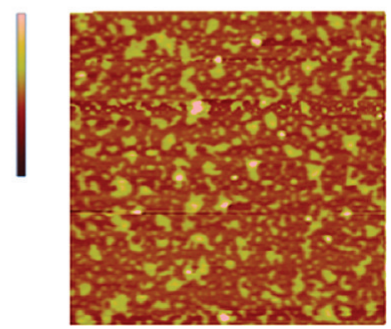

Figure 9. AFM images of PLL cushioned lipid membrane containing (A) 0.5 mol \% DSPE-PEG 2000 , (B) $1.5 \mathrm{~mol}_{\%}$ DSPE-PEG 2000 ,

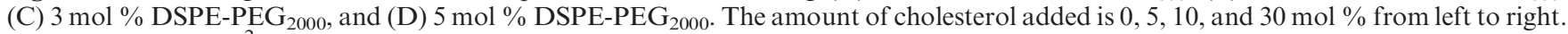
Scan size is $5 \times 5 \mu \mathrm{m}^{2}$.

although the whole quality of membranes is still incompetent for application. Otherwise, some of PLL cushioned phospholipid membranes with $30 \mathrm{~mol} \%$ cholesterol incorporation present accumulating lipid islands, which make the surfaces look much rougher. It might be caused by the complexes formation of lipids and cholesterol. ${ }^{37,38}$ Comparing with the relatively smooth and uniform dried lipid membrane on chitosan, even heavy cholesterol loading can only leave scattering membrane debris on the PLLmodified substrate. From this view, the properties of membrane supporting polymers, such as electrostatic force, wettability, and roughness, are also important factors for the final structure of lipid membrane in air.

\section{Conclusion}

In summary, we have developed a facile method for the formation of air-stable phospholipid membranes on silicon

(37) Engelman, D. M.; Rothman, J. E. J. Biol. Chem. 1972, 247, 3694.

(38) Radhakrishnan, A.; Li, X. M.; Brown, R. E.; McConnell, H. M. Biochim. Biophys. Acta 2001, 1511, 1-6. dioxide surface, which is confirmed by imaging ellipsometry and AFM. Hydrophilic polysaccharide cushion and PEG shielding together function in maintaining air stability of lipid membrane. The density of DSPE-PEG 2000 is a critical parameter in the final structure of dry lipid membrane, and only a proper content of PEGylated lipids addition can help form air-stable lipid membrane. Taking the advantage of this air-stable platform, we have confirmed the condensing and ordering effect of cholesterol on lipid membrane.

Acknowledgment. We thank Mr. Liang Tian of Jinan Haidebei Marine Bioengineering Co. for kindly gifting chitosan and Public Center of Suzhou Institute of Nano-Tech and NanoBionics for AFM characterization and spectroscopy ellipsometry measurement. The authors gratefully acknowledge financial support from Ministry of Science and Technology of China (2008AA02Z419), Natural Science Foundation (10804083, 20845003) of China, Chinese Academy of Sciences (KJCX2. YW.M04), and Key Laboratory of Microgravity, Institute of Mechanics, CAS. 